CERN-TH.6852/93

\title{
Multiscalar amplitudes to all orders in perturbation theory
}

\author{
E.N. Argyres

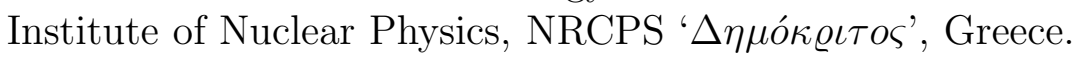 \\ Ronald H.P. Kleiss, \\ NIKEHF-H, Amsterdam, the Netherlands \\ Costas G. Papadopoulos \\ TH Division, CERN, Geneva, Switzerland
}

\begin{abstract}
A method for calculating loop amplitudes at the multiboson threshold is presented, based on Feynman-diagram techniques. We explicitly calculate the one-loop amplitudes in both $\phi^{4}$-symmetric and broken symmetry cases, using dimensional regularization. We argue that, to all orders in the perturbation expansion, the unitarityviolating behaviour of the tree-order amplitudes persists.
\end{abstract}

CERN-TH.6852/93

March 1993 
The high-multiplicity limit of processes involving scalar particles has recently come under investigation [1, 2]. At tree order, the cross section grows as $n$ !, where $n$ is the number of produced scalar particles, and it violates the unitarity bound for sufficiently high energies. The construction of an effective potential that respects the unitarity bound is possible [3]. In order to make this effective theory consistent at any $n$ and for any energy, derivative coupling must be added, which generally leads to a complicated theory.

One-loop amplitudes have been calculated [4, 5] recently. The method used in these calculations is based on the fact that the generating function of one-loop threshold amplitudes is obtained by expanding the field around the classical background (which is the generating function of tree-order threshold amplitudes) and keeping the first term [6]. More recently Voloshin [7] argued that in broken symmetry $\phi^{4}$ theory, the factorial growth of the amplitude, which is related to the radius of convergence of the generating function, persists to all orders in perturbative expansion. In this letter we develop a method for calculating loop amplitudes at threshold based on Feynman diagrams, in analogy with the tree-order calculations [2]. We start with the $\phi^{4}$ theory. The Lagrangian is given by

$$
\mathcal{L}=\frac{m^{2}}{2} \phi^{2}+\frac{\lambda}{24} \phi^{4}
$$

The recursion relation for the one-loop amplitude is represented diagrammatically in Fig.11. It is of the form

$$
\begin{aligned}
\frac{a_{1}(n)}{n !}= & -i \frac{\lambda}{2} \sum \frac{i a_{1}\left(n_{1}\right)}{n_{1} !\left(n_{1}^{2}-1\right)} \frac{i a\left(n_{2}\right)}{n_{2} !\left(n_{2}^{2}-1\right)} \frac{i a\left(n_{3}\right)}{n_{3} !\left(n_{3}^{2}-1\right)} \\
& -i \frac{\lambda}{2} \mu^{2 \epsilon} \sum \frac{i a\left(n_{1}\right)}{n_{1} !\left(n_{1}^{2}-1\right)} \int \frac{d^{D} k}{(2 \pi)^{D}} \frac{D\left(n_{2} ; k\right)}{n_{2} !} \\
& -i T_{2} \frac{i a(n)}{n !\left(n^{2}-1\right)}-i \frac{T_{4}}{6} \sum \frac{i a\left(n_{1}\right)}{n_{1} !\left(n_{1}^{2}-1\right)} \frac{i a\left(n_{2}\right)}{n_{2} !\left(n_{2}^{2}-1\right)} \frac{i a\left(n_{3}\right)}{n_{3} !\left(n_{3}^{2}-1\right)}
\end{aligned}
$$

where $a_{1}(n)$ is the one-loop amplitude and $a(n)$ the tree-order one. The integral over $k$ extends over the Minkowski space. Throughout this paper we assume that $m=1$ and we restore the mass dependence when it is necessary. The counterterms contributions are

$$
\begin{aligned}
& T_{2}=\frac{1}{2} m^{2} \frac{\lambda}{16 \pi^{2}}\left(\frac{1}{\epsilon}+b\right) \\
& T_{4}=\frac{3}{2} \lambda \frac{\lambda}{16 \pi^{2}}\left(\frac{1}{\epsilon}+c\right)
\end{aligned}
$$

where $b$ and $c$ are in principle, arbitrary constants used to define the renormalization conditions. $D(n ; k)$ is the propagator of the field with the emission of $n$ particles [ [4] (see Fig.尹). It satisfies the following recursion relation:

$$
\frac{D(n ; k)}{n !}=\frac{1}{\left[(k+n q)^{2}-1+i \varepsilon\right]} \frac{\lambda}{2} \sum \frac{i a\left(n_{1}\right)}{n_{1} !\left(n_{1}^{2}-1\right)} \frac{i a\left(n_{2}\right)}{n_{2} !\left(n_{2}^{2}-1\right)} \frac{D\left(n_{3} ; k\right)}{n_{3} !}
$$



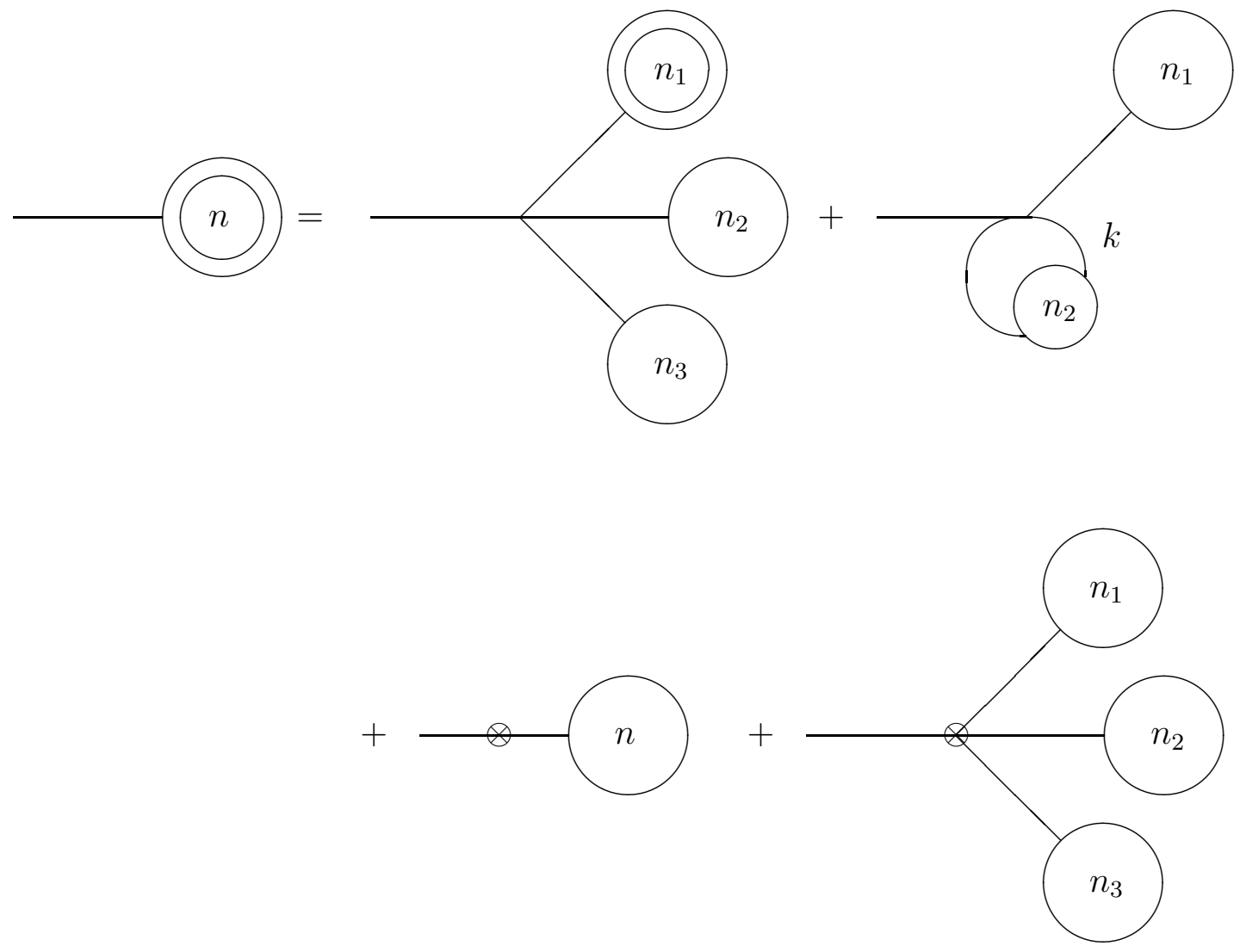

Figure 1: Diagrammatic representation of the recursion formula for the one-loop amplitudes $\mathcal{A}(1 \rightarrow n)$ (blob with two circles). The blobs with on circle connected to a line are the tree-order amplitude $\mathcal{A}(1 \rightarrow n)$. The blob with one circle connected to two lines corresponds to the propagator with the emission of $n$ particles.

Making the ansatz $D(n ; k)=i n ! d(n)$ and defining $g(x)=\sum d(n) x^{n}$, we have

$$
\left(x^{2} \frac{d^{2}}{d x^{2}}+(2 k \cdot q+1) x \frac{d}{d x}+k^{2}-1+i \varepsilon-\frac{\lambda}{2} \phi_{0}^{2}\right) g=1
$$

where

$$
\phi_{0}=\frac{x}{1-\frac{\lambda}{48} x^{2}}
$$

is the generating function of tree-order amplitudes [8]. Under the substitutions $x=i \sqrt{48 / \lambda} e^{\tau}, u=e^{\tau}, \omega=\sqrt{\vec{k}^{2}+1-i \varepsilon}, \epsilon=k \cdot q$ and $g=e^{-\epsilon \tau} y(\tau)$ we obtain

$$
\left(\frac{d^{2}}{d \tau^{2}}-\omega^{2}+\frac{6}{\cosh ^{2}(\tau)}\right) y=e^{\epsilon \tau} .
$$




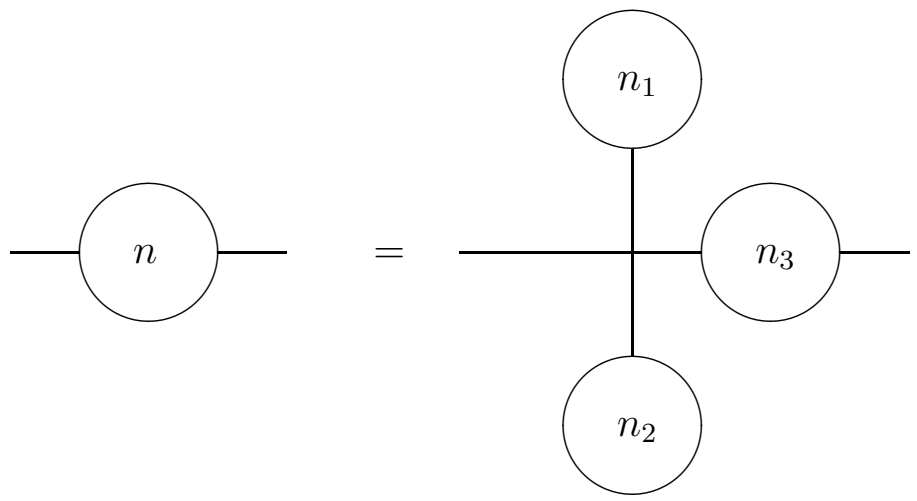

Figure 2: Diagrammatic representation of the recursion relation for the propagator with the emission of $n$ particles.

The solutions of the homogeneous part are

$$
\begin{aligned}
& f_{1}=\frac{(2-\omega)(1-\omega)+2 u^{2}\left(\omega^{2}-4\right)+u^{4}(2+\omega)(1+\omega)}{u^{\omega}\left(1+u^{2}\right)^{2}} \\
& f_{2}=f_{1}(\omega \rightarrow-\omega) .
\end{aligned}
$$

The Wronskian is $W=2 \omega\left(\omega^{2}-1\right)\left(\omega^{2}-4\right)$ and the solution for $g(\tau)$ is given by

$$
g(\tau)=-\frac{e^{-\epsilon \tau}}{W}\left(f_{1}(\tau) \int_{-\infty}^{\tau} d s e^{\epsilon s} f_{2}(s)+f_{2}(\tau) \int_{\tau}^{\infty} d s e^{\epsilon s} f_{1}(s)\right)
$$

Taking into account the results for $a(n)$ and $D(n ; k)$ we arrive at the following differential equation:

$$
\left(\frac{d^{2}}{d \tau^{2}}-1-\frac{\lambda}{2} \phi_{0}^{2}\right) \phi_{1}=-\frac{\lambda}{2} \phi_{0} \int \frac{d^{D} k}{(2 \pi)^{D}} g(\tau ; k)+T_{2} \phi_{0}+\frac{T_{4}}{6} \phi_{0}^{3}
$$

where $\phi_{1}$ is the generating function of 1-loop amplitudes, $\phi_{1}=\sum b_{1}(n) x^{n}$ and $a_{1}(n)=-i n ! b_{1}(n)$. The integral over $k$ now runs over the Euclidean space.

The integration over $k^{0}$ gives

$$
\mu^{2 \epsilon} \int \frac{d^{D} k}{(2 \pi)^{D}} g(\tau ; k)=-\mu^{2 \epsilon} \int \frac{d^{d} k}{(2 \pi)^{d}} \frac{f_{1} f_{2}}{W}
$$

where $d=D-1$. It is straightforward to see that

$$
\frac{f_{1} f_{2}}{W}=\frac{1}{2\left(\vec{k}^{2}+1\right)^{\frac{1}{2}}}\left(1-\frac{\lambda}{4} \phi_{0}^{2} \frac{1}{\vec{k}^{2}}+\frac{\lambda^{2}}{16} \phi_{0}^{4} \frac{1}{\vec{k}^{2}\left(\vec{k}^{2}-3-i \varepsilon\right)}\right) .
$$

In dimensional regularization, the first two terms give poles $\frac{1}{\epsilon}$, whereas the third term is finite, having an imaginary part coming from the infrared singularity at 
$\omega=2 m$. The final result can be written as

$$
\left(\frac{d^{2}}{d \tau^{2}}-1-\frac{\lambda}{2} \phi_{0}^{2}\right) \phi_{1}=B \phi_{0}+C \phi_{0}^{3}+F \phi_{0}^{5}
$$

where

$$
\begin{aligned}
B & =\frac{m^{2}}{2} \frac{\lambda}{16 \pi^{2}}\left(b+\gamma_{E}-1-\log \left(\frac{4 \pi \mu^{2}}{m^{2}}\right)\right) \\
C & =\frac{\lambda}{4} \frac{\lambda}{16 \pi^{2}}\left(c+\gamma_{E}-2-\log \left(\frac{4 \pi \mu^{2}}{m^{2}}\right)\right) \\
F & =-\frac{\lambda^{3} \sqrt{3}}{1536 \pi^{2}}\left(\log \frac{2+\sqrt{3}}{2-\sqrt{3}}-i \pi\right)
\end{aligned}
$$

and $\gamma_{E}$ is the Euler-Mascheroni constant. Assuming, for instance, that $B=0$, we find that the general solution to Eq.(14) is given by

$$
\phi_{1}=\frac{3 C}{\lambda}\left(\frac{d}{d \tau} \phi_{0}-\phi_{0}\right)+F \frac{\lambda^{3}}{24} \frac{x^{5}}{\left(1-\frac{\lambda}{48} x^{2}\right)^{3}} .
$$

The term multiplying $F$ has also been given in ref. [⿴囗⿴囗十).

We can repeat the same analysis in the case of the spontaneously broken $\phi^{4}$ theory. The Lagrangian, after shifting, is given by

$$
\mathcal{L}=\frac{m^{2}}{2} \phi^{2}+\frac{m \sqrt{3 \lambda}}{6} \phi^{3}+\frac{\lambda}{24} \phi^{4}
$$

and the one-loop generating function satisfies the equation

$$
\begin{aligned}
\left(\frac{d^{2}}{d \tau^{2}}-1-\sqrt{3 \lambda} \phi_{0} \frac{\lambda}{2} \phi_{0}^{2}\right) \phi_{1}= & -\left(\frac{\sqrt{3 \lambda}}{2}+\frac{\lambda}{2} \phi_{0}\right) \int \frac{d^{D} k}{(2 \pi)^{D}} g(\tau ; k) \\
& +T_{1}+T_{2} \phi_{0}+\frac{T_{3}}{2} \phi_{0}^{2}+\frac{T_{4}}{6} \phi_{0}^{3} .
\end{aligned}
$$

The counterterms are given by

$$
\begin{aligned}
& T_{1}=\frac{\sqrt{3 \lambda} m^{3}}{32 \pi^{2}}\left(\frac{1}{\epsilon}+c_{1}\right) \\
& T_{2}=\frac{\lambda m^{2}}{8 \pi^{2}}\left(\frac{1}{\epsilon}+c_{2}\right) \\
& T_{3}=\frac{3 \lambda m \sqrt{3 \lambda}}{32 \pi^{2}}\left(\frac{1}{\epsilon}+c_{3}\right) \\
& T_{4}=\frac{3 \lambda^{2}}{32 \pi^{2}}\left(\frac{1}{\epsilon}+c_{4}\right)
\end{aligned}
$$

Performing the $k^{0}$ integration we get

$$
\mu^{2 \epsilon} \int \frac{d^{D} k}{(2 \pi)^{D}} g(\tau ; k)=-\mu^{2 \epsilon} \int \frac{d^{d} k}{(2 \pi)^{d}} \frac{f_{1} f_{2}}{W}
$$


where

$$
\frac{f_{1} f_{2}}{W}=\frac{1}{2\left(\vec{k}^{2}+1\right)^{\frac{1}{2}}}\left(1+\frac{3 u}{(1+u)^{2}} \frac{1}{\left(\vec{k}^{2}+\frac{3}{4}\right)}+\frac{9 u^{2}}{(1+u)^{4}} \frac{1}{\left(\vec{k}^{2}+\frac{3}{4}\right) \vec{k}^{2}}\right) .
$$

Note that we can take the limit $i \varepsilon \rightarrow 0$ : this means that the amplitude does not develop an imaginary part, as pointed out in refs. [8, 5].

The final result is now written in the form

$$
\begin{aligned}
\left(\frac{d^{2}}{d \tau^{2}}-1-\sqrt{3 \lambda} \phi_{0} \frac{\lambda}{2} \phi_{0}^{2}\right) \phi_{1}= & \left(\frac{\sqrt{3 \lambda}}{2}+\frac{\lambda}{2} \phi_{0}\right) \frac{\sqrt{3}}{2 \pi} \frac{u^{2}}{(1+u)^{4}} \\
& +C_{1}+C_{2} \phi_{0}+C_{3} \phi_{0}^{2}+C_{4} \phi_{0}^{3}
\end{aligned}
$$

where

$$
\begin{aligned}
& C_{1}=\frac{\sqrt{3 \lambda}}{32 \pi^{2}}\left(c_{1}+\gamma_{E}-\log \left(\frac{4 \pi \mu^{2}}{m^{2}}\right)-1\right) \\
& C_{2}=\frac{\lambda}{16 \pi^{2}}\left(2\left[c_{2}+\gamma_{E}-\log \left(\frac{4 \pi \mu^{2}}{m^{2}}\right)\right]-\frac{7}{2}+\frac{\sqrt{3}}{2} \pi\right) \\
& C_{3}=\frac{3 \lambda \sqrt{3 \lambda}}{64 \pi^{2}}\left(c_{3}+\gamma_{E}-\log \left(\frac{4 \pi \mu^{2}}{m^{2}}\right)-2+\frac{\sqrt{3}}{3} \pi\right) \\
& C_{4}=\frac{\lambda^{2}}{64 \pi^{2}}\left(c_{4}+\gamma_{E}-\log \left(\frac{4 \pi \mu^{2}}{m^{2}}\right)-2+\frac{\sqrt{3}}{3} \pi\right)
\end{aligned}
$$

Taking $c_{i}$ such that $C_{i}=0$, we find that

$$
\phi_{1}=\frac{\sqrt{\lambda}}{2 \pi} \frac{u^{2}}{\left(1+u^{3}\right)}
$$

in agreement with [5].

The previous calculations can be extended to higher orders in perturbation theory. In order to proceed, we have to add recursively new counterterms to the original Lagrangian, in such a way that we keep its form invariant. This can be done (say for the $\phi^{4}$-symmetric case) by expressing, as usual, the bare constants in terms of the renormalized ones:

$$
\begin{aligned}
\lambda_{0} & =\mu^{2 \epsilon}\left(c_{0}\left(\lambda, \frac{\mu}{m}, \epsilon\right)+\sum_{k=1}^{\infty} \frac{c_{k}\left(\lambda, \frac{\mu}{m}\right)}{\epsilon^{k}}\right) \\
m_{0}^{2} & =m^{2}\left(b_{0}\left(\lambda, \frac{\mu}{m}, \epsilon\right)+\sum_{k=1}^{\infty} \frac{b_{k}\left(\lambda, \frac{\mu}{m}\right)}{\epsilon^{k}}\right) \\
Z_{\phi} & =\left(\zeta_{0}\left(\lambda, \frac{\mu}{m}, \epsilon\right)+\sum_{k=1}^{\infty} \frac{\zeta_{k}\left(\lambda, \frac{\mu}{m}\right)}{\epsilon^{k}}\right)
\end{aligned}
$$

where $b_{0}, c_{0}, \zeta_{0}$ are analytic as $\epsilon \rightarrow 0$.

Let $a_{L}(n)$ being the $L^{\text {th }}$-order amplitude for the production of $n$ scalars at threshold and $\phi_{L}$ the corresponding generating function. The equation it satisfies is given by

$$
\left(\frac{d^{2}}{d \tau^{2}}-1-\frac{\lambda}{2} \phi_{0}^{2}\right) \phi_{L}=\lambda \phi_{L-1} \phi_{1} \phi_{0}+\ldots+\mathcal{F}+T_{2}^{(L)} \phi_{0}+\frac{T_{4}^{(L)}}{6} \phi_{0}^{3}
$$


where $T_{2}^{(L)}$ and $T_{4}^{(L)}$ are the corresponding $L^{\text {th }}$-order counterterms, whose finite part as $\epsilon \rightarrow 0$ is in general arbitrary (renormalization-scheme-dependent) and corresponds to the definition of the $L^{\text {th }}$-order $\mathcal{A}(1 \rightarrow 1)$ and $\mathcal{A}(1 \rightarrow 3)$ amplitudes. The ellipsis corresponds to lower-loop contributions and $\mathcal{F}$ represents all explicit $L$-loop graphs (in analogy to the one-loop case).

Assuming now that the high- $n$ behaviour is independent of the renormalization prescription, i.e. the way we define the parameters $b_{0}, c_{0}$ and $\zeta_{0}$ in the counterterm Lagrangian, it is easy to see that the leading behaviour of the solution $\phi_{L}$ should be more singular at $\tau= \pm i \frac{\pi}{2}\left(x \equiv x_{0}=\sqrt{\frac{48}{\lambda}}\right)$ than the combination $\frac{d}{d \tau} \phi_{0}-\phi_{0}$ (see Eq.(18)), since we can compensate any change in the coefficient multiplying $\phi_{0}^{3}$ in Eq. (28), by adding to the solution $\phi_{L}$ a term proporional to $\frac{d}{d \tau} \phi_{0}-\phi_{0}$. In general equating the most singular terms in Eq.(28) at $x=x_{0}$, we have that

$$
\rho(L)+2=\rho(L-1)+\rho(1)+1, L \geq 2
$$

where $-\rho(L)$ is the singularity-exponent at $x_{0}$. Barring accidental cancellations, whose origin should be traced in a rather non-trivial symmetry, a solution to Eq.(29) is of the form $\rho(L)=2 L+1$. Using the above result we obtain that, to all orders in perturbation theory the large- $n$ behaviour, which is connected with the radius of convergence of the generating function, is given by $\sim n !\left(n^{2} \lambda\right)^{L}$ for both the unbroken and the broken symmetry cases. We find ourselves in agreement with [7], where the same conclusion has been drawn but from a different point of view.

We conclude that the amplitudes $\mathcal{A}\left(H^{*} \rightarrow n H\right)$ exhibit a high- $n$ behaviour, which leads to unitarity violation for all loops. Still, there is the possibility that summing up all the perturbation series one gets a consistent result. Nevertheless, if this happens, it relies on an essentially non-perturbative phenomenon. Having proven that, for scalar amplitudes, perturbation theory breaks down in the large- $n$ limit, we can speculate that either a non-perturbative phenomenon takes place at high energies, leading for instance to a redefinition of the multi-Higgs states, in analogy with the QCD confinement, or a symmetry should exist which kills the factorial behaviour at threshold and hopefully generates unitarity-respecting cross sections, in analogy with gauge-symmetries and tree-order high-energy behaviour [9]. 


\section{References}

[1] M.B. Voloshin, Nucl.Phys. B383 (1992) 233.

[2] E.N. Argyres, R. Kleiss and C.G. Papadopoulos, Nucl.Phys.B391 (1993) 42.

[3] E.N. Argyres, R. Kleiss and C.G. Papadopoulos, Phys.Lett.B296 (1992) 139.

[4] M.B. Voloshin, 'Summing one-loop graphs at multiparticle threshold', Univ. of Minnesota preprints TPI-MINN-92/45-T and 'Comment on 'Summing oneloop graphs at multi-particle threshold", TPI-MINN-92/46-T.

[5] B.H. Smith, 'Summing one-loop graphs in a theory with broken symmetry', Univ. of Minnesota preprint TPI-MINN-92/50-T.

[6] L.S. Brown, Phys.Rev.D46 (1992) R4215.

[7] M.B. Voloshin, 'Some properties of amplitudes at multi boson thresholds in spontaneously broken scalar theory', Univ. of Minnesota preprint TPI-MINN92/61-T.

[8] E.N. Argyres, R. Kleiss and C.G. Papadopoulos, 'Multiscalar production amplitudes beyond threshold', CERN-TH.6629/92.

[9] J.M. Cornwall, D.N. Levin and G. Tiktopoulos, Phys. Rev. D10 (1974), 1145. 